BULL. AUSTRAL. MATH. SOC.

VOL. $23(1981), 161-179$.

\title{
SOME RESULTS FOR THE \\ GENERAL BULK SERVICE QUEUEING SYSTEM
}

D.F. Holman, M.L. Chaudhry and A. Ghosal

\begin{abstract}
This paper deals with a general bulk service queueing system with one server, for which customers arrive in a Poisson stream and the service is in bulk. The maximum number of customers to be served in one lot is $B$ (capacity), but the server does not start service until $A$ (quorum, less than $B$ ) customers have accumulated; and the service time follows a general probability distribution. Probability generating functions of the distributions $P_{n}$ and $P_{n}^{+}$, under equilibrium, have been derived by using the supplementary variable technique. An expression has been derived for the expected value of the queue size and its relation with the expected value of the waiting time of a customer has been explored. A numerical case has been worked out on the assumption that bulk service follows a specified Erlangian distribution.
\end{abstract}

\section{Introduction}

In real life we come across queueing situations in which service is rendered in bulk. A general bulk-service system with single server is such that the server, when he is free, starts service only if a specified minimum, say $A$, of customers have accumulated in the queue and he does not take more than $B$ customers for service in one lot. The paper

Received 5 September 1980. The research for this paper was supported (in part) by the CRAD, Grant Number 3610-603. Part of the work was done, by $A$. Ghosal, at the Royal Military College, Kingston, Ontario, on leave during summer 1980 . 
discusses a system for which the customers arrive in a Poisson stream (in other words, inter-arrival times between customers follow an exponential distribution with mean, say $1 / \lambda)$, and the batch service time follows a general probability distribution function, say $B(x)$, with a mean service rate $\mu$. Let such a system be called $M / G^{A, B} / 1$. (If the inter-arrival time had followed a general probability distribution function, the system would have been called $G I / G^{A, B} / 1$.)

If there is no quorum (that is, if $A=1$ ), the system described above reduces to one discussed by Bailey [1], it will be referred to as the $M / G^{B} / 1$ system. A system in which both $A$ and $B$ are equal will be referred to as an $M / G^{A} / 1$ system - it was discussed by Fabens [8], amongst others. The $M / G^{A, B} / 1$ system was dealt with first by Neuts [17].

Various workers, apart from the authors referred to above, have derived probability distribution of the queue size under equilibrium condition for various descriptions of bulk queues - see Bloemena [3], Jaiswal [12], [13], Foster and Nyunt [9], Foster and Parera [10], Chaudhry and Templeton [4], and so on. (References to relevant work have been given at the end of this paper.) Most of them attempted to derive the generating function of either of the two probabilities under equilibrium state, as given below:

$P_{n}^{+}$equals the probability that the number in queue just after a departure epoch is $n(n=0,1,2, \ldots)$,

$P_{n}$ equals the probability that the number in queue at a random epoch is $n(n=0,1,2, \ldots)$.

In queueing literature, $P_{n}$ is equivalent to the probability that a customer on arrival finds the queue size to be $n$ (under equilibrium condition). The approach of most of the authors (see Foster and Parera [10], Fabens [8]) has been: firstly to derive $P_{n}^{+}$or its generating function $P^{+}(z)\left(=\sum_{n=0}^{\infty} P_{n}^{+} z^{n}\right)$ and then to relate it to $P_{n}$ or its generating function $P(z)\left(=\sum P_{n} z^{n}\right)$ by using the theory of semi-Markov 
processes or the renewal theory.

In this paper the technique of supplementary variables has been used to derive the generating function $P(z)$ directly for $M / G^{A, B} / 1$ system; this technique was used by Chaudhry and Templeton [4] for a simpler bulk service system. Neuts [17] had obtained an exact solution for this system by using semi-Markov process, but the results were not computationally tractable. The method used here enables one to derive the expected value and the variance of the queue size in a simpler way. This has been elucidated for an $M / E_{k}^{A, B} / 1$ system, in which the service time follows an Erlangian distribution.

A contribution of this paper has been to relate $\left\{P_{n}\right\}$ of the system to its $\left\{P_{n}^{+}\right\}$, where $P_{n}^{+}$has been derived by using the imbedded Markov chain technique.

Finally the relation between the expected value of the queue size and the expected value of the waiting time has been explored.

\section{Equilibrium distribution of number in queue}

Let $N_{q}(t)$ be the queue size (defined here to denote the number of customers waiting for service, not including those in service) and $X(t)$ the elapsed service time of the group undergoing service at time $t$. The supplementary variable $X(t)$ is introduced in order to obtain a bivariate Markov process $\left\{N_{q}(t), X(t)\right\}$. Let us now define the following joint probabilities :

$$
\begin{aligned}
v_{r}(t) & \equiv \operatorname{Prob}\left(N_{q}(t)=r \text { and the server is idie }\right), 0 \leq r<A, \\
u_{r}(t) & \equiv \operatorname{Prob}\left(N_{q}(t)=r \text { and the server is busy }\right), 0 \leq r, \\
u_{r}(x, t) & \equiv \lim _{\Delta x \rightarrow 0}\left[\operatorname{Prob}\left(N_{q}(t)=r, x<X(t) \leq x+\Delta x\right) / \Delta x\right], r \geq 0 .
\end{aligned}
$$

In limiting case, that is, when $t \rightarrow \infty$, the argument $t$ in the above functions is suppressed. We may also note the following relations:

$$
\begin{aligned}
& v_{i}=0 \text { if } i \geq A \text { or } i<0 \text {; } \\
& u_{i}=0 \text { if } i<0 \text {; }
\end{aligned}
$$




$$
u_{i}(x)=0 \text { if } i<0 \text {; }
$$

and

$$
u_{p}=\int_{0}^{\infty} u_{p}(x) d x
$$

We also define the conditional service rate, $n(x)$, conditional that service has not yet been completed at time $x$ :

$$
\eta(x)=b(x) /(1-B(x)),
$$

so that $b(x)$ and $B(x)$ are respectively density function and distribution function of the service time, given by

$$
\begin{aligned}
& b(x)=n(x) \exp \left\{-\int_{0}^{x} n(t) d t\right\}, \\
& B(x)=1-\exp \left\{-\int_{0}^{x} n(t) d t\right\} .
\end{aligned}
$$

Following Cox [5] or Chaudhry and Templeton [4], we may now apply the supplementary variable technique to the problem by forming the equations of the state of the system in steady state. They are

$$
\lambda v_{0}=\int_{0}^{\infty} u_{0}(x) n(x) d x,
$$

$$
\lambda v_{r}-\lambda v_{r-1}=\int_{0}^{\infty} u_{r}(x) \eta(x) d x, \quad 1 \leq r \leq A-1,
$$

$$
\begin{aligned}
& \frac{\partial}{\partial x} u_{0}(x)=-[\lambda+n(x)] u_{0}(x), \\
& \frac{\partial}{\partial x} u_{n}(x)=-[\lambda+n(x)] u_{n}(x)+\lambda u_{n-1}(x), n>0 .
\end{aligned}
$$

These equations are to be solved subject to the following boundary conditions:

$$
u_{0}(0)=\sum_{r=A}^{B} \int_{0}^{\infty} u_{r}(x) \eta(x) d x+\lambda v_{A-1},
$$

$$
u_{n}(0)=\int_{0}^{\infty} u_{n+B}(x) n(x) d x, n>0
$$


Next we define the following probability generating functions:

$$
\begin{gathered}
u(z) \equiv \sum_{n=0}^{\infty} u_{n} z^{n}, \\
u(x, z) \equiv \sum_{n=0}^{\infty} u_{n}(x) z^{n} .
\end{gathered}
$$

Let us define

$$
u(z)=\int_{0}^{\infty} u(x, z) d x
$$

We also define $P(z)$ to be the probability generating function of the number in the queue, regardless of whether the server is busy or not. Obviously, then the following is true:

$$
P(z)=u(z)+\sum_{i=0}^{A-1} v_{i} z^{i}
$$

We now proceed to develop an expression for $u(z)$. Multiplying equation (6) by $z^{n}$ summing from $n=1$ to infinity and adding equation (5), we get a differential equation which on solution gives

$$
u(x, z)=u(0, z) e^{-(\lambda-\lambda z) x}[1-B(x)],
$$

where we have used the relation (2).

We next find $u(0, z)$ from the boundary conditions, as follows. Using equation ( 3$),(4),(7)$ and $(8)$, one can get after some simplification (11) $u(0, z)=z^{-B} \sum_{r=0}^{B}\left(z^{B}-z^{p}\right) \int_{0}^{\infty} u_{r}(x) \eta(x) d x+z^{-B} \int_{0}^{\infty} u(x, z) n(x) d x$. After using (10) and the definition of $\eta(x)$, we get after simplification

$$
u(0, z)=\left(\sum_{r=0}^{B}\left(z^{B}-z^{r}\right) \int_{0}^{\infty} u_{p}(x) n(x) d x\right) /\left(z^{B}-\bar{b}(\lambda-\lambda z)\right) .
$$

Next, substitution of (12) in equation (10) gives 
(13) $u(x, z)=\left(\left(\sum_{r=0}^{B}\left(z^{B}-z^{r}\right) \int_{0}^{\infty} u_{r}(y) n(y) d y\right) /\left(z^{B}-\bar{b}(\lambda-\lambda z)\right)\right)$ $\times e^{-(\lambda-\lambda z) x_{1}}[1-B(x)]$.

Recall now that $u(z)=\int_{0}^{\infty} u(x, z) d x$. The equation (13) can be so integrated, using one step of integration by parts, to give

$$
u(z)=u(0, z) \cdot(1-\bar{b}(\lambda-\lambda z)) /(\lambda-\lambda z) .
$$

This is the probability generating function of number in the queue with the server busy. Further discussion of this will be taken up in a later section. It is of considerable interest to note that the above expression has no dependence on $A$, the quorum. It agrees exactly with the similar expression found by Chaudhry and Templeton [4] for the system $M / G^{B} / 1$.

\section{Relation to the imbedded Markov chain}

Next we wish to relate the probability generating function in equation (14) to $P^{+}(z)$, the probability generating function of the number in the system immediately after departure epochs or equivalently just before commencement of service epochs:

$$
P^{+}(z)=\sum_{i=0}^{\infty} P_{i}^{+} z^{i} \text {, where } P_{i}^{+} \text {probability that the number is } i .
$$

This procedure is new and has been used for the first time by Chaudhry and Templeton [4] for analyzing bulk service queues. For computational results, this allows us to use the steady-states imbedded Markov chain probabilities, which can be calculated as shown below. We note from Chaudhry and Templeton [4] that $P_{n}^{+}$is related to $u_{n}(x)$ as follows:

$$
P_{n}^{+}=d \int_{0}^{\infty} u_{n}(x) n(x) d x
$$

where $d$ is a normalizing constant. The equation (15) is based on the definitions of $u_{n}(x)$ and $n(x)$. The probability generating function $P^{+}(z)$ can be seen to be 


$$
P_{n}^{+}(z)=d \int_{0}^{\infty} u(x, z) n(x) d x
$$

Using equation (10), we get

$$
P^{+}(z)=d u(0, z) \bar{b}(\lambda-\lambda z),
$$

where we have used the definition of $\eta(x)$. Now taking $u(0, z)$ from equation (12) we get

$$
P^{+}(z)=d\left(\left[\sum_{r=0}^{B}\left(z^{B}-z^{r}\right) \int_{0}^{\infty} u_{p}(x) n(x) d x\right) /\left(z^{B}-\bar{b}(\lambda-\lambda z)\right)\right) \cdot \bar{b}(\lambda-\lambda z) .
$$

Using equation (15) this simplifies to

$$
P^{+}(z)=\left(\left[\sum_{r=0}^{B}\left(z^{B}-z^{r}\right) P_{r}^{+}\right) /\left(z^{B}-\bar{b}(\lambda-\lambda z)\right)\right] \cdot \vec{b}(\lambda-\lambda z) .
$$

It needs to be pointed out here that equation (18) may be compared with equation (12) of Neuts' paper [17] with $H_{i}(\cdot)=H(\cdot)$, $i=0,1,2, \ldots, B$.

Again it is interesting to note that there is no dependence on $A$, the quorum. Thus, this expression holds for the imbedded Markov chain results for $M / G^{A} / 1$ and $M / G^{B} / 1$; and indeed we note exact agreement with expressions for these systems developed by Takács [20], Foster and Nyunt [9], and Chaudhry and Templeton [4]. It appears that although the three systems $M / G^{A} / 1, M / G^{B} / 1$ and $M / G^{A, B} / 1$ have different underlying processes, these differences do not surface at the instants examined by the imbedded Markov chain. Thus, whether the server is intermittently available (Bailey's model), or waits for a customer when he is idle and serves him as soon as he arrives (Jaiswal or Bloemena or Chaudhry's model) or waits for $A$ customers when he is idle (Foster and Nyunt or Takács' model), the imbedded Markov chain results are the same. In his paper concerning the $M^{X} / G^{Y} / 1$ system $\left(M^{X}\right.$ implies that the arrival process is also a Poisson stream but $X$ people can arrive in bulk at a given epoch), Bhat [2] has raised this same observation; however, he had not considered the present $M / G^{A, B} / 1$ system. Some details of Bhat's paper may be found in Prabhu [19]. We can evaluate the constant $d$ by applying the 
normalizing condition, $P^{+}(1)=1$, to equation (16).

We find

$$
d=[u(0,1)]^{-1}
$$

We evaluate $[u(0,1)]^{-1}$ from equation (14), by applying the normalizing condition to this equation, recalling from equation (9) that

$$
u(1)=1-\sum_{r=0}^{A-1} v_{r} \text {, the total probability that the server is busy. }
$$

Finally, we get, using L'Hôpital's rule,

$$
u(0,1)=\mu\left[1-\sum_{r=0}^{A-1} v_{r}\right] \text {, }
$$

a result which is intuitively appealing and may be obtained by the conservation principle. This gives

$$
d=\left[\mu\left(1-\sum_{r=0}^{A-1} v_{r}\right)\right]^{-1}
$$

By comparing equations (14) and (17), and using (20) we get the relation

$$
\mu(z) / P^{+}(z)=(1-\bar{b}(\lambda-\lambda z)) /(\lambda-\lambda z) \cdot\left(\mu\left(1-\sum_{r=0}^{A-1} v_{r}\right)\right) / \bar{b}(\lambda-\lambda z) .
$$

We may also point out here that the probability generating function $P^{-}(z)$ for the number in the queue just before arrival epochs is simply the same as $P(z)$ due to the characteristics of the Poisson arrival process.

\section{Calculation of basic $P_{r}^{+}$and $v_{i}$ probabilities}

We next discuss the calculation of the unknown probabilities, $P_{r}^{+}$, $r=0,1, \ldots, B$ and then these are related to the idle-server probabilities, $v_{i}, i=0,1, \ldots, A-1$.

The characteristic equation of this queueing system is, from the denominator of (18),

$$
z^{B}-\vec{b}(\lambda-\lambda z)=0
$$


The equation (22) has $B$ zeros inside and on $C$ (for details, see Foster and Nyunt [9]). There is a simple zero at $z=1$, and therefore, there are $B-1$ other zeros inside the unit circle. Let us call them $z_{i}, i=1,2, \ldots, B-1$.

Consider again the equation (18). Since it must converge inside and on the unit circle, the numerator must vanish at all the zeros of the denominator which are inside and on the unit circle. The zero at $z=1$ clearly cancels from both numerator and denominator, leaving $B-1$ other equations :

$$
\sum_{r=0}^{B-1}\left(z_{i}^{B}-z_{i}^{r}\right) P_{r}^{+}=0, \quad i=1,2, \ldots, B-1 .
$$

To this we add the normalization condition, $P^{+}(1)=1$, which gives

$$
\sum_{r=0}^{B-1}(B-r) P_{r}^{+}=B(1-\rho) \text {. }
$$

Together, the system of equations (23) and (24) form a set of $B$ linearly independent equations in the $B$ unknowns $P_{r}^{+}$. Therefore, the $P_{\gamma}^{+}$can be found from the zeros of the characteristic equation inside and on the unit circle. Thus the probability generating function $P^{+}(z)$ is completely determined. We now wish to relate the $P_{r}^{+}$'s to the $v_{i}^{\prime} s$.

Recall the equation (15), and using the value of $d$ from (20), we have

$$
\int_{0}^{\infty} u_{n}(x) n(x) d x=\mu\left(1-\sum_{i=0}^{A-1} v_{i}\right) P_{n}^{+} .
$$

This relationship is substituted into equations (3) and (4) giving

$$
\begin{aligned}
\lambda v_{0} & =\mu\left(1-\sum_{i=0}^{A-1} v_{i}\right) P_{0}^{+}, \\
\lambda v_{r}-\lambda v_{r-1} & =\mu\left(1-\sum_{i=0}^{A-1} v_{i}\right) P_{r}^{+}, 1 \leq r \leq A-1 .
\end{aligned}
$$

Multiplying (25) by $A$ and adding to it the equation (26) multiplied by $(A-r)$ and summed over $r=1$ to $A-1$, we get an equation which gives 
(27)

$$
I-\sum_{i=0}^{A-1} v_{i}=(\lambda / \mu) /\left(\lambda / \mu+\sum_{r=0}^{A-1}(A-r) P_{r}^{+}\right) .
$$

Since $P^{+}(z)$ is already fully determined, (27) together with the equation (2l) is sufficient to give $u(z)$ completely. However, it is still necessary to find values of individual $v_{i}$ 's to use in (9). First, substituting (27) into (25) gives,

$$
v_{0}=P_{0}^{+} /\left(\lambda / \mu+\sum_{r=0}^{A-1}(A-r) P_{r}^{+}\right) \text {. }
$$

Also summing (25) and (26) for $r=1$ to $n$ gives an equation which, with the use of (27) gives

$$
v_{n}=\left(\sum_{r=0}^{n} P_{r}^{+}\right) /\left(\lambda / \mu+\sum_{r=0}^{A-1}(A-r) P_{r}^{+}\right), \quad 0 \leq n \leq A-1 .
$$

We now wish to evaluate the term $\sum_{i=0}^{A-1} v_{i} z^{i}$ to complete the determination of $P(z)$ in equation (9). From (29), we get

$$
\left.\sum_{i=0}^{A-1} v_{i} z^{i}=\int_{r=0}^{A-1}\left(z^{r}-z^{A}\right) P_{r}^{+}\right) /\left[\left[\lambda / \mu+\sum_{r=0}^{A-1}(A-r) P_{r}^{+}\right](1-z)\right) \text {. }
$$

Finally, this gives the expression for $P(z)$ :

$$
\left.P(z)=u(z)+\left(\sum_{r=0}^{A-1}\left(z^{r-z^{A}}\right) P_{r}^{+}\right) /\left(\left[\lambda / \mu+\sum_{r=0}^{A-1}(A-r) P_{r}^{+}\right](1-z)\right)\right) \text {. }
$$

5. Expected number in queue and expected waiting time in queue The expected number in the queue, $L_{q}$, is found as usual by taking the first derivative of $(30)$ and setting $z=1$. The derivative of the first term, $u^{\prime}(1)$, is found from (21), which on using (27), gives

$$
u^{\prime}(1)=\left((\lambda / \mu) /\left(\left[\lambda / \mu+\sum_{r=0}^{A-1}(A-r) P_{r}^{+}\right]\right)\right]\left[L^{+}+(\lambda \mu / 2)\left(\sigma^{2}-\left(1 / \mu^{2}\right)\right)\right] \text {, }
$$

where $L^{+}=P^{+}(1)$ and $\sigma^{2}$ is the variance of the service time distribution given by $\bar{b}^{\prime \prime}(0)=\sigma^{2}+\left(1 / \mu^{2}\right)$. 
The derivative of the second term is

$$
\left(\left(\frac{1}{2}\right) \sum_{r=0}^{A-1}[A(A-1)-r(r-1)] P_{r}^{+}\right) /\left(\lambda / \mu+\sum_{r=0}^{A-1}(A-r) P_{r}^{+}\right) .
$$

Combining terms, we get

$$
\begin{aligned}
L_{q}=(1 /(\lambda / \mu & \left.\left.+\sum_{r=0}^{A-1}(A-r) P_{r}^{+}\right)\right) \\
& \times\left\{(\lambda / \mu) L^{+}+\left(\lambda^{2} / 2\right)\left(\sigma^{2}-\left(1 / \mu^{2}\right)\right)+\frac{1}{2} \sum_{r=0}^{A-1}[A(A-i)-r(r-1)] P_{r}^{+}\right\} .
\end{aligned}
$$

Thus $L_{q}$ is determined in terms of $L^{+}$and the $P_{r}^{+}$'s. The latter can be found, as described earlier, from the roots of the characteristic equation. Similarly $L^{+}$can be found by expressing $P^{+}(z)$ in terms of the roots of the characteristic equation and differentiating, as usual.

While discussing the waiting time distribution for the queueing system $M / M^{A, B} / 1$, Medhi [16] reports that the Little's formula $L_{q}=\lambda W_{q}$ (where $W_{q}$ is the expected waiting time in the queue) holds true. It is conjectured by the authors that this relation should hold true for $M / G^{A, B} / 1$ case also or even for $G I / G^{A, B} / 1$ system, asymptotically. A combinatorial argument may be developed on the following lines.

For a $G I / G / 1$ system, let

$B_{k}(n)$ equal the probability that the $n$th customer on starting his service finds $k$ customers waiting behind,

$w_{n}$ equal the waiting time of the $n$th customer,

$t_{n}$ equal the time interval between arrivals of the $n$th and $(n+1)$ th customers.

Then

$$
\sum_{k=i}^{\infty} H_{k}(n)=\operatorname{Prob}\left(w_{n}>t_{n}+t_{n+1}+\ldots+t_{n+i-1}\right) .
$$

By replacing $t_{\dot{r}}$ by its expected value $(r=n, \ldots, n+i-1) 1 / \lambda$, we get, on some general conditions, 


$$
\sum_{k=i}^{\infty} H_{k}(n) \rightarrow \operatorname{Prob}\left(w_{n}>i / \lambda\right)
$$

Summing over $i$, for large $n$, we get (since $w_{n}$ follows a continuous distribution function)

$$
\begin{aligned}
\sum_{i=1}^{\infty} \sum_{k=i}^{\infty} H_{k}(n) & =\sum_{i} \operatorname{Pr}\left(\omega_{n}>k / \lambda\right) \\
& +\lambda \int_{0+}^{\infty} \operatorname{Pr}(\omega>x) d x,
\end{aligned}
$$

whence

$$
L_{q} \rightarrow \lambda W_{q}
$$

Since, for an $M / G^{A, B} / 1$ system, $\sum_{r=n}^{n+i-1} t_{r}$ in (33) does not depend on $A$ or $B$, the result (34) should hold good asymptotically. In the asymptotic case, $P_{k}^{-}$approximates $H_{k}(n)$.

A rigorous proof of (34) will be published by the authors in a subsequent communication.

\section{Expected busy and idle periods}

In this section we determine the expected values of the busy and idle periods using zero-one process. This technique can be applied to the present system in a straight-forward fashion, and yields the expected value directly.

Let $\eta$ and $I$ be random variables which represent respectively the busy and idle times of the server. Accordingly, using the zero-one process (Parzen [18]) we get the relationship

$$
\frac{E(\eta)}{E(I)}=\lim _{t \rightarrow \infty} \frac{\operatorname{Prob}(\text { server is busy at time } t)}{\text { Prob(server is idle at time } t)} .
$$

The probability that the server is idle in equilibrium is given by the sum $\sum_{i=0}^{A-1} v_{i}$. Therefore (35) becomes 


$$
\frac{E(\eta)}{E(I)}=\left(1-\sum_{i=0}^{A-1} v_{i}\right) /\left(\sum_{i=0}^{A-1} v_{i}\right)
$$

We next proceed to evaluate $E(I)$. There are $A$ states of the system with the server idle: those states with $0,1,2, \ldots, A-1$ customers in the queue and the server is idle. Thus the total probability that the server is idle just after a service departure is $\sum_{r=0}^{A-1} P_{r}^{+}$. Therefore the probability of the server being idle with $n$ customers in the queue on a service departure is

$$
P_{n}^{+} /\left(\sum_{r=0}^{A-1} P_{r}^{+}\right), 0 \leq n \leq A-1 \text {. }
$$

If there are $n$ in the queue on a service departure, which begins an idle period, there must be $A-n$ units that arrive before the idle period ends. Accordingly, the expected value of such an idle period is $(A-n) / \lambda$, and the overall expected value is

$$
E(I)=\left(\sum_{n=0}^{A-I}(A-n) P_{n}^{+}\right) /\left(\lambda \sum_{r=0}^{A-1} P_{r}^{+}\right) .
$$

It should be noted that this expected value as stated above is conditional that the idle period is non-zero.

Now continuing (36) and (37) we get the expected busy period:

$$
E(n)=\left(\sum_{n=0}^{A-1}(A-n) P_{n}^{+}\right) /\left(\lambda \sum_{r=0}^{A-1} P_{r}^{+}\right) \cdot\left(1-\sum_{i=0}^{A-1} v_{i}\right) /\left(\sum_{i=0}^{A-1} v_{i}\right) .
$$

Using equation (27) this can be simplified to give

$$
E(\eta)=\left[\mu \sum_{r=0}^{A-1} P_{r}^{+}\right]^{-1} .
$$

The special cases of (36) with $B=A$, or $A=1$ and $G=E_{R}$, where $E_{R}$ is a modified Erlang distribution, have been discussed by Fabens [8] and Jaiswal [14], respectively. 


\section{The queue $M / E_{k}^{A, B} / 1$ - a particular case}

By specifying a service time distribution we can discuss some further results of this system. Since the k-Erlang distribution $\left(b(x)=\left\{(\mu k)^{k} /(k-1) !\right\} \exp (-\mu k x) x^{k-1}, \mu>0, k \geq 1, x \in[0, \infty)\right)$ is the most general and will be a good approximation to systems with service time distributions whose coefficient of variation is less than 1 , this is the one we investigate.

In this case $\bar{b}(s)=[\mu /(\mu+\varepsilon)]^{k}$. Therefore the characteristic equation becomes

$$
z^{B}=[\mu /(\mu+\lambda-\lambda z)]^{k}
$$

Following Bailey [1], it can be shown that

$$
P^{+}(z)=\prod_{i=1}^{k}\left(\theta_{i}-1\right) /\left(\theta_{i}-z\right)
$$

where $\theta_{i}, i=1,2, \ldots, k$, are the zeros outside the unit circle.

This is the same equation reported by Bailey for the system $M / E_{k}^{B} / I$, but also applies to our more general case. Thus the expected number in the system at departure epochs is easily found to be

$$
L^{+}=P^{+},(1)=\sum_{i=1}^{k}\left(\theta_{i}-1\right)^{-1} \text {. }
$$

Moreover, partial fraction expansion of (40) allows us to find expressions for the $P_{n}^{+}$'s in terms of roots $\theta_{i}$. This gives

$$
P_{n}^{+}=\sum_{i=1}^{k}\left(1-\theta_{i}^{-1}\right) / \theta_{i}^{n} \prod_{\substack{j=1 \\ j \neq i}}^{k}\left(\theta_{j}-1\right) /\left(\theta_{j}-\theta_{i}\right) \text {. }
$$

This is an alternative approach to find the $P_{n}^{+}$'s from the method described in Section 4. It allows us, then, to calculate the $v_{i}$ probabilities, and along with $L^{+}$from (41) gives all the information needed to find $L_{q}$ from (32), $E(I)$ from (37) and $E(n)$ from (38). 


\section{Numerical calculations for $M / E_{k}^{A, B} / 1$}

Some typical numerical calculations of the $P_{n}^{+}$and $v_{i}$ probabilities and the expected values $L^{+}$and $L_{q}$ were undertaken by the authors using The Royal Military College PDP-11/34 computer. We use equation (42) for the $P_{n}^{+}$, (29) for $v_{i},(41)$ for $L^{+}$, and (32) for $L_{q}$.

The preliminary step, then, was to find the $\theta_{i}$, the roots of equation (37) with modulus greater than unity. It was found more convenient here to change variable setting $x=1 / z$ and then finding the roots inside the unit circle. These roots were then inverted to give the desired $\theta_{i}$ 's . Bairstow's method of extracting pairs of roots, which are in general complex, was used with considerable success. All other manipulations of these roots involved complex arithmetic, a requirement which caused no real difficulty. When probabilities were calculated it was noted that the values obtained were pure real numbers within the tolerance of the round-off errors of the computer.

Extensive work concerning the numerical evaluation of the roots of (37) appears to have been undertaken by Downton [6], [7], Kotiah, et al [15], amongst others. Now efficient computer algorithms are available to solve such polynomials. Indeed, in another connection Grassman [11] has shown that it takes only a few seconds on a computer to find the roots of a polynomial of degree as far as 50 . As such no attempt was made here to reproduce large numerical data. However, we did undertake numerical evaluation of various expected values for a few values of the parameters involved. The results were quite encouraging. The case when $\rho=0.9$, is presented below. The tables give various expected values in units of the mean service time for $\rho=0.9, B=5, A=1(1) 5, K=1(1) 3$.

A remark regarding some numerical calculations may be in order here. Jaiswal gives a table of numerical values for the mean busy-period for the case when $P(R=1)=1$. In some cases, his values differ from the ones found by us in the second decimal place. For example, if the busy-period is computed in terms of the mean interarrival time as done by Jaiswal, then for $\rho=.9, B=5, E(n)=128.877$ in our case, but it is 128.809 in Jaiswal's case. 


$$
M / E_{k}(A, B) / 1
$$

TABLE OF VALUES OF $L_{q}$

$$
\rho=0.9, B=5
$$

\begin{tabular}{c|ccccc}
${ }_{k}^{A}$ & 1 & 2 & 3 & 4 & 5 \\
\hline 1 & 27.426 & 27.017 & 26.451 & 25.768 & 25.009 \\
2 & 18.643 & 18.196 & 17.493 & 16.610 & 15.645 \\
3 & 15.749 & 15.259 & 14.433 & 13.382 & 12.272
\end{tabular}

TABLE OF VALUES OF $E(\eta)$

$$
\rho=0.9
$$

\begin{tabular}{l|crrrr}
$k$ & 1 & 2 & 3 & 4 & 5 \\
\hline 1 & 9.999 & 14.659 & 19.319 & 23.979 & 28.639 \\
2 & 4.756 & 7.181 & 10.185 & 13.756 & 17.896 \\
3 & 3.025 & 4.268 & 5.938 & 8.084 & 19.761
\end{tabular}

TABLE OF VALUES OF $E(I)$

$$
\rho=0.9
$$

\begin{tabular}{c|ccccc}
${ }^{B}$ & 1 & 2 & 3 & 4 & 5 \\
\hline 1 & 1.111 & .844 & .754 & .709 & .687 \\
2 & 1.111 & .798 & .694 & .634 & .600 \\
3 & 1.111 & .778 & .660 & .599 & .561
\end{tabular}

\section{Concluding remarks}

The problem of obtaining the probability distribution of the queue size in a general bulk service queue with Poisson arrival stream $\left(M / G^{A, B} / 1\right)$ has been dealt with in this paper. A contribution of this paper is to relate the probability generating function of the queue size at random point (that is, queue length when a customer arrives at a random 
point of time) to the probability generating function of the queve size left on a departure epoch by a batch served; such a relation has been given in the equation (30). Difficulty of deriving such a relationship for his general model was pointed out by Neuts [17]. The system discussed here is somewhat less general than the one which was considered by Neuts but has the advantage of being amenable to easy calculations regarding the expected value and standard deviation of the queue size. The queueing system

$M / E_{k}^{A, B} / 1$ has been worked out with numerical calculations. Finally a discussion has been made on the applicability of Little's relation between the expected value of the queue size and the expected value of the waiting time in an $M / G^{A, B} / 1$ system.

\section{References}

[1] Norman T.J. Bailey, "On queueing processes with bulk service", $J$. Roy. Statist. Soc. Ser. B 16 (1954), 80-87.

[2] U. Narayan Bhat, "Imbedded Markov chain analysis of single server bulk queues", J. Austral. Math. Soc. 4 (1964), 244-263.

[3] A.R. Bloemena, "On queueing processes with a certain type of bulk service", Bull. Inst. Internat. Statist. 37 (1960), 219-226.

[4] M.L. Chaudhry and J.G.C. Templeton, "The queueing system $M / G^{B} / 1$ and its ramifications", European J. Oper. Res. 6 (1981), 56-60.

[5] D.R. Cox, "The analysis of non-Markovian stochastic processes by the inclusion of supplementary variables", Proc. Cambridge Philos. Soc. 51 (1955), 433-441.

[6] F. Downton, "On limiting distributions arjsing in bulk service queues", J. Roy. Statist. Soc. Ser. B 18 (1956), 265-274.

[7] F. Downton, "Waiting time in bulk service queues", J. Roy. Statist. Soc. Ser. B 17 (1955), 256-261.

[8] Augustus J. Fabens, "The solution of queueing and inventory models by semi-Markov processes", J. Roy. Statist. Soc. Ser. B 23 (1961), 113-127. 
[9] F.G. Foster and K.M. Nyunt, "Queues with batch departures I", Ann. Math. Statist. 32 (1961), 1324-1332.

[10] F.G. Foster and A.G.A.D. Perera, "Queues with batch departures II", Ann. Math. Statist. 35 (1964), 1147-1156.

[11] Winfried Grassman, Some computational aspects of the $M / E_{k} / 1$ queue in steady state (Mimeograph Series 449. Department of Statistics, Purdue University, 1976).

[12] N.K. Jaiswal, "Bulk-service queueing problem", Oper. Res. 8 (1960), 139-143.

[13] N.K. Jaiswal, "Time-dependent solution of the bulk-service queueing problem", Oper. Res. 8 (1960), 773-781.

[14] N.K. Jaiswal, "Distribution of busy periods for the bulk-service queueing problem", Defence Sci. J. 12 (1962), 309-316.

[15] T.C.T. Kotiah, J.W. Thompson, W.A.O'N. Waugh, "Use of Erlagian distributions for single-server queueing systems", J. Appl. Prob. 6 (1969), 584-593.

[16] J. Medhi, "Waiting time distribution in a Poisson queue with a general bulk service rule", Management Sci. 21 (1974/1975), $777-782$.

[17] Marcel F. Neuts, "A general class of bulk queues with Poisson input", Ann. Math. Statist. 38 (1967), 759-770.

[18] Emanual Parzen, Stochastic processes (Holden-Day, San Francisco, London, Amsterdam, 1967).

[19] N.U. Prabhu Queues and inventories. A study of their basic stochastic processes (John Wiley \& Sons, New York, London, Sydney, 1965). 
[20] Lajos Takács, Introduction to the theory of queues (Oxford University Press, New York, 1962).

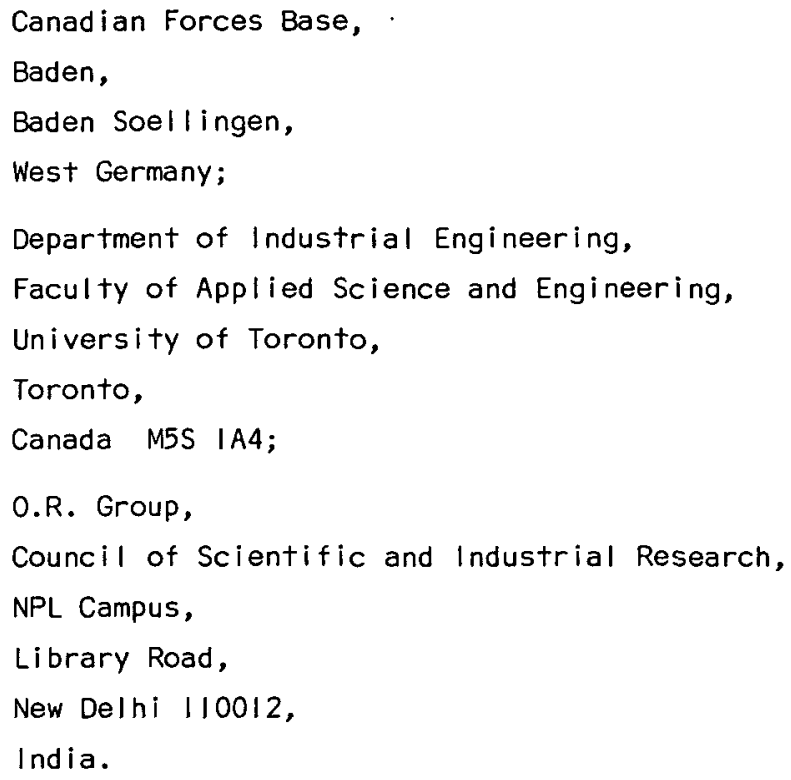

